\title{
Modeling of specific structure crystallization coupling with dissolution
}

\author{
Yuanhui JI ${ }^{1}$, Hongliang QIAN ${ }^{1}$, Chang LIU ${ }^{1}$, Xiaoyan $\mathrm{JI}^{2}$, Xiaohua LU (ه) ${ }^{1}$, Xin FENG ${ }^{1}$ \\ 1 State Key Laboratory of Materials-Oriented Chemical Engineering, Nanjing University of Technology, Nanjing 210009, China \\ 2 Division of Energy Engineering, Luleå University of Technology, 97187 Luleå, Sweden
}

(C) Higher Education Press and Springer-Verlag Berlin Heidelberg 2010

\begin{abstract}
In this paper, the research framework for specific structure crystallization modeling has been proposed in which four steps are required in order to investigate the rigorous crystallization modeling by thermodynamics. The first is the activity coefficient model of the solution, the second is Solid-Liquid equilibrium, the third and fourth are the dissolution and crystallization kinetics modeling, respectively. Our investigations show that the mechanisms of complex structure formation and microphase transition can be analyzed by combining the dissolution and crystallization kinetics modeling. Moreover, the formation mechanism of the porous $\mathrm{KCl}$ has been analyzed, which may provide a reference for the porous structure formation in the advanced material synthesis.
\end{abstract}

Keywords activity coefficient model, solid-liquid equilibrium, dissolution kinetics, crystallization modeling, porous structures, microphase separation

\section{Introduction}

What are the frontier and challenges in chemical engineering nowadays? As we know, in the past century, chemical engineering has played a very important role in fertilizer, petroleum industry, etc. In the new century, product engineering has been proposed by Prof. James Wei [1] to investigate the relationships between the structures and the performances of the materials. In China, multiscale method [2] has been proposed by Prof. Jinghai Li and his co-workers. With the development of science and technology, the interdisciplinary research between chemical engineering and material science has been more and

Received November 2, 2009; accepted November 30, 2009

E-mail: xhlu@njut.edu.cn more important. Therefore, the new discipline of materialsoriented chemical engineering [3] has come out. For the new discipline of materials-oriented chemical engineering [3], new advanced materials are used to develop new chemical processes, and chemical engineering methods are applied to the material engineering. Thus, the objective of our laboratory is to prepare the advanced inorganic materials and some nanomaterials. Our targets are the same as chemical engineering processes, that is, large-scale preparation, high quality with low cost, and the process should be controlled easily. However, if we deal with the solid, it is quite different from the previous chemical engineering processes. As we know from phase diagram to chemical process simulation, thermodynamics has played a very important role in the petroleum industry. However, if the system has become more complex, including complex interface, complex structures, and complex facets, how do we deal with it by thermodynamics? This is one challenge for us. For our objective, firstly, we should do some experiments in the laboratory, and this means advanced material synthesis; secondly, we need the crystallization modeling, and this modeling should explain both mechanism and morphology of the crystals. After the working on both objectives, we can establish the scale-up method, and it means we should find some controllable operation parameters. This is what chemical engineering can do for the new solid materials. In this work, the research framework for crystallization modeling is proposed in Fig. 1. The chemical potential can be used to judge which process will occur. If the chemical potential of the liquid equals that of the solid, it is in the equilibrium, but this equilibrium is different from the vapour-liquid (VL) equilibrium. For the VL systems, only two phases - vapour and liquid - exist. However, if we deal with the solid, we may have much more phases. Therefore, the solid-liquid equilibrium (SLE) calculation is also the challenge. If the chemical potential of the liquid is different from that of the solid, either dissolution or crystallization 
will occur. The $\Delta \mu$ is the driving force for either the dissolution or the crystallization process. From the chemical potential, at least four steps should be performed for specific structure crystallization modeling and will be introduced with inorganic salts as the study examples in this paper.

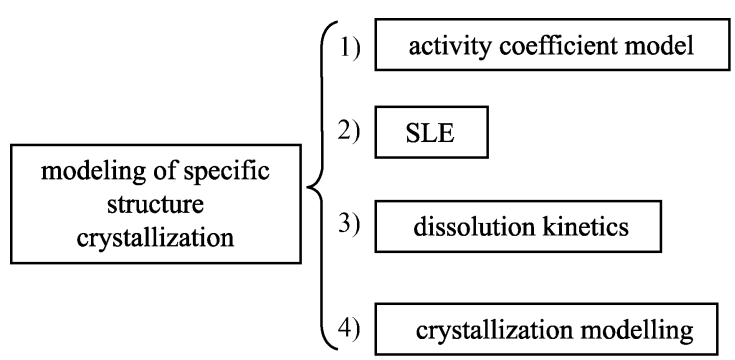

Fig. 1 Research framework for specific structure crystallization modeling

\section{Thermodynamic modeling}

2.1 Activity coefficient model in mixed aqueous electrolyte solutions

The first step is activity coefficient model in mixed aqueous electrolyte solutions. There are growing interests in thermodynamic properties of mixed aqueous electrolyte solutions due to the importance for understanding various natural and industrial processes such as inorganic chemical manufacture, wastewater treatment, seawater desalination, etc. The existing models for describing and correlating activity coefficients in aqueous electrolyte solutions are roughly assigned to physical or chemical models. Most activity coefficient models are only appropriate for single aqueous electrolyte solutions, and their extensions to mixed electrolyte solutions are often not reliable enough, particularly at high ionic strength near the solubility limit.

In our research group, a new model (Lu-Maurer model) [4] for describing activity coefficient in mixed aqueous electrolyte solutions is proposed, which combines chemical reaction (ionic solvation equilibria) with physical interactions (local composition equation). This model describes the Gibbs excess energy, especially osmotic coefficient $\phi$ and mean activity coefficients $\gamma_{ \pm}$of dissolved electrolytes in an aqueous solution. Dissolving a strong electrolyte in water results in a mixture of water molecules, unsolvated, and solvated ions. Incomplete dissociation and ion pairing are both neglected. The true concentrations of solvated and unsolvated ions from the overall concentration of the dissolved electrolytes are calculated from solvation equilibria. Deviation from ideal mixing behavior is considered by combining the DebyeHückel law with the UNIQUAC model. The new method is especially aimed to predicting properties in aqueous mixed electrolyte solutions from the properties of single aqueous electrolyte solutions. By introducing some reasonable assumptions, the model parameters can be reduced to three "pure component" parameters per ion and two binary parameters for interactions between a cation and an anion from experimental results of single aqueous electrolyte systems. Parameters are determined for 10 cations $\left(\mathrm{H}^{+}, \mathrm{Li}^{+}, \mathrm{Na}^{+}, \mathrm{K}^{+}, \mathrm{Cs}^{+}, \mathrm{NH}_{4}^{+}, \mathrm{Mg}^{2+}, \mathrm{Ca}^{2+}, \mathrm{Sr}^{2+}\right.$, and $\left.\mathrm{Ba}^{2+}\right)$ and 6 anions $\left(\mathrm{Cl}^{-}, \mathrm{Br}^{-}, \mathrm{I}^{-}, \mathrm{OH}^{-}, \mathrm{NO}_{3}^{-}\right.$, and $\left.\mathrm{SO}_{4}^{2-}\right)$ at $298.15 \mathrm{~K}$. The capabilities of the new model for predicting properties of mixed aqueous electrolyte solutions are investigated by comparing predicted osmotic coefficients, mean ionic activity coefficients, and solubilities of salts with experimental results and predictions by the standard method of Pitzer (1973), and some of its modifications for not only dilute but also concentrated mixed aqueous electrolyte solutions at $298.15 \mathrm{~K}$.

Moreover, the model proposed by Lu and Maurer [4] for mixed aqueous electrolyte solutions is extended to predict the activity coefficients from $273 \mathrm{~K}$ to temperatures up to $573 \mathrm{~K}[5,6]$. The extension is achieved by introducing a universal relation for the influence of temperature on some model parameters. Although the parameters are ion specific, the influence of the temperature on those parameters is universal. No additional parameters are required for describing mixed aqueous electrolyte solutions. The model accurately predicts activity coefficients at high temperatures and concentrations up to solubility limit in aqueous electrolyte solutions containing, for example, $\mathrm{K}^{+}, \mathrm{Na}^{+}, \mathrm{NH}_{4}^{+}, \mathrm{SO}_{4}^{2-}, \mathrm{Cl}^{-}$, and $\mathrm{NO}_{3}^{-}[5,6]$.

\subsection{SLE stage in aqueous electrolyte solutions}

The second step is the SLE stage in aqueous electrolyte solutions. Processes related to aqueous electrolyte solutions and solids are important in areas such as inorganic chemicals, materials science, biochemistry, environmental protection, etc. In the related systems, electrolytes dissociate completely or partly into cations and anions, solid phases are immiscible, and compounds are formed in the different solid phase. The number of phases and their identities are too complicated to predict, which causes difficulties for the design of such processes.

The number of phases and their identities can be determined in advance using phase diagrams or the enumerative method during process design. The former method is unsuitable for complicated systems and cannot calculate continuously. In the latter method, all combinations should be tried, and good initial values are often required to calculate the activity coefficients reliably. The difficulty of estimating initial values limits the wide use of this method in practice.

For another method based on free energy minimization, the number of phases and their identities must be determined in advance for free energy calculation. Presently, this is studied by the enumerative method. 
Furthermore, the calculation of the free energy is related to activity coefficients. For aqueous electrolyte solutions, many models have been proposed, and the reliability of the model for calculating activity coefficients extends to saturated solutions. To calculate activity coefficients reliably and obtain a reliable free energy, a good initial estimate is required to ensure that the composition in liquid phase is within or near saturated conditions. For the important need of crystallization process simulations, one must predict whether a given feed will be split into multiple phases, the number of phases and their identities at equilibrium, and the distribution of components within these phases (the SLE stage). Moreover, the proposed method should be generalized, reliable, and computationally efficient. To satisfy these requirements, a general method for the SLE stage is proposed in our group [7] in which the number of phases and their identities at equilibrium are determined automatically in advance.

On the basis of quantity of solvent in the feed and the calculated intervals, the proposed method is much simpler and more computationally efficient than the enumerative method as the solvent is considered as a single and independent variable. Furthermore, good initial estimates and increments are considered carefully to ensure that the solution is within or near the saturated range. Meanwhile, the proposed method for the SLE stage is used to predict crystallization paths and generate phase diagrams of SLE. It can also be added to the commercial software Aspen Plus or Pro/II to simulate processes involving solid phases. Several cases are examined to demonstrate the capability of this method for use with systems of hydrate, multi-ion, and complex salts [7].

\subsection{Dissolution kinetics modeling of $\mathrm{K}_{2} \mathrm{SO}_{4}$ crystal}

The third step is the rigorous dissolution kinetics modeling of crystals. Dissolution of solids in solutions has widespread applications in oceanography, crystallography, metallurgy, ceramics, and desalination technology as well as in many biological and environmental precipitation processes [8]. Concentration measurement by sampling with a regular interval [9] or by the atomic absorption [8] cannot be performed online. ISEs are effective to obtain basic and reliable experimental data for dissolution process investigations [10].

In our work [11], the dissolution kinetics of $\mathrm{K}_{2} \mathrm{SO}_{4}$ crystals is studied and the effects of hydrodynamic situation and temperature on the dissolution are investigated with ISEs. The dissolution is determined by measuring the change of electromotive force $(E)$ with potassium ion selective electrode. From the values of $E$, the concentrations of $\mathrm{K}^{+}$are calculated combined with an activity coefficient model. The results are accurate and rapid, and the maximum deviation is less than $2 \%$. Theory, developed for constant bulk concentration in a rotating disk system, is modified to analyze the general grain dissolution process. Normal distribution is introduced in the crystal area calculation in order to obtain reliable dissolution rate. In our work, the statistical rate theory is modified to analyze the experimental data in the general grain dissolution or growth process. In our modification, undersaturation is based on activities instead of concentrations, and the calculations of equilibrium exchange rate $k_{\mathrm{e}}$ and thickness of diffusion layer are derived. With the determined experimental data and modified theory, diffusion rate constant, equilibrium exchange rate constant, and thickness of diffusion layer are obtained. As expected, diffusion rate constant increases with the increase of temperature and stirring speed, the equilibrium exchange rate is dependent on bulk concentration, and the thickness of diffusion layer depends on both temperature and stirring speed. Furthermore, the effect of temperature on the thickness of diffusion layer is more than that of stirring speed.

Moreover, the dissolution kinetics of $\mathrm{K}_{2} \mathrm{SO}_{4}$ crystal in aqueous ethanol solutions is also studied online with ISEs [12]. The concentration of $\mathrm{K}_{2} \mathrm{SO}_{4}$ is calculated from the determined electromotive force in which the activity coefficient of components in liquid phase is calculated with the Pitzer model. Dissolution kinetics parameters in the modified statistical rate theory are regressed. The correlation results show that dissolution rate of $\mathrm{K}_{2} \mathrm{SO}_{4}$ is slower in aqueous ethanol solutions than that in aqueous solutions. The two most important reasons are 1) the solubility of $\mathrm{K}_{2} \mathrm{SO}_{4}$ in aqueous ethanol solutions is lower than that in aqueous solutions, which causes a decrease of driving force of diffusion, and 2) the surface reaction of $\mathrm{K}_{2} \mathrm{SO}_{4}$ becomes slower due to the addition of ethanol, so the dissolution process is mainly controlled by the surface reaction instead of diffusion.

\subsection{Crystal growth kinetics modeling of $\mathrm{K}_{2} \mathrm{SO}_{4}$}

The fourth step is the rigorous crystal growth kinetics modeling of crystals. Crystallization has been widely used for separation and purification including inorganic salts and proteins $[13,14]$. Because crystallization temperature shows an important effect on the solubility of solute, product yield, and crystallization rate, it is one of the key parameters for design of both crystallizers and crystallization processes [14].

Generally, the crystal growth rate increases with increasing temperatures. However, the temperature dependence of the crystal growth rate of $\mathrm{K}_{2} \mathrm{SO}_{4}$ is abnormal in the low temperature range; that is, the growth rate decreases with increasing temperatures from $273.15 \mathrm{~K}$ to $293.15 \mathrm{~K}$. This abnormal phenomenon is probably due to the deposition of fine crystals on the seed crystal surface, which may enhance the crystal growth rate [15]. Theoretical investigations are needed to explain it clearly.

On the other hand, the solution is complicated for the large-scale production of $\mathrm{K}_{2} \mathrm{SO}_{4}$. For example, if $\mathrm{K}_{2} \mathrm{SO}_{4}$ is 
produced from $\mathrm{Na}_{2} \mathrm{SO}_{4}$ and $\mathrm{KCl}$, the solution contains $\mathrm{Na}^{+}, \mathrm{K}^{+}, \mathrm{Cl}^{-}$, and $\mathrm{SO}_{4}^{2-}$ during the crystallization process [16]. The complicated solution may cause complexity of the temperature effect on the growth rate of $\mathrm{K}_{2} \mathrm{SO}_{4}$ and the difficulty in theoretical study.

Most of the previous studies are based on empirical equations to correlate experimental data, and the driving force of crystallization is simplified to be the concentration difference between the crystal and the bulk solution. However, the fundamental driving force for crystallization is the chemical potential difference of crystal at the solidliquid interface $[17,18]$ in which a thermodynamic model is required to calculate activity coefficients and chemical potential. The rigorous theoretical investigations on crystallization are carried out based on chemical potential difference.

In our previous introduction, thermodynamic properties of aqueous electrolyte solutions are studied. In our work [19], the temperature dependence of the crystal growth rate of $\mathrm{K}_{2} \mathrm{SO}_{4}$ in aqueous $\mathrm{K}_{2} \mathrm{SO}_{4}$ solutions and $\mathrm{KCl}-\mathrm{K}_{2} \mathrm{SO}_{4}$ solutions is investigated.

The crystal growth rate equation of $\mathrm{K}_{2} \mathrm{SO}_{4}$ is developed with chemical potential difference of $\mathrm{K}_{2} \mathrm{SO}_{4}$ at the solidliquid interface as the basic driving force of crystallization. The Lu-Maurer model [4] is used to predict the activity coefficient of $\mathrm{K}_{2} \mathrm{SO}_{4}$ in aqueous $\mathrm{K}_{2} \mathrm{SO}_{4}$ and $\mathrm{KCl}-\mathrm{K}_{2} \mathrm{SO}_{4}$ solutions. The crystal growth rate of $\mathrm{K}_{2} \mathrm{SO}_{4}$ in aqueous $\mathrm{K}_{2} \mathrm{SO}_{4}$ solution decreases and then increases with increasing temperature and reaches its lowest value at $303.15 \mathrm{~K}$, which is in accordance with previous experimental results.

\section{Thermodynamic modeling of porous structure formation from phase transition coupling with dissolution}

After the above four steps have been achieved, we will focus on some specific crystals. As we know, porous materials have been widely used because of their important applications: biomaterials, separation systems, catalyst, etc. Therefore, in this work, we will focus on the porous crystals. However, how is the porous structure formed? In our work, carnallite is selected as an example. When carnallite dissolves in solutions, $\mathrm{KCl}$ will recrystallize, and porous structured $\mathrm{KCl}$ with a pore size of $10-50$ microns is produced from the fractional crystallization of carnallite [20]. Moreover, Fick's second law is used to investigate the diffusion of $\mathrm{K}^{+}, \mathrm{Mg}^{2+}$, and $\mathrm{Cl}^{-}$, and the ionic product of carnallite and $\mathrm{KCl}$ is analyzed to explain the formation mechanism of the porous $\mathrm{KCl}$ [20]. However, in the fractional crystallization of carnallite, during the diffusion of ions, the dissolution or crystallization may occur at a certain position inside carnallite crystals. In order to analyze the formation mechanism of porous structures further and then to provide a reference for the porous structure formation, a model for diffusion coupling with dissolution and crystallization is proposed in which the driving force is described with the potential gradient.

In the model, ion diffusion and crystal crystallization/ dissolution are considered, and the fluid flow is neglected. The ion diffusion begins from the left boundary, and dissolution or crystallization may occur at a certain position inside the channels. The positive chemical potential difference $(\Delta \mu)$ means that the aqueous solutions are supersaturated and crystallization may occur, the negative $\Delta \mu$ means that the aqueous solution is unsaturated or crystal may dissolve, and the zero value of $\Delta \mu$ means that the aqueous solution is saturated and neither crystallization nor dissolution will occur.

The ion diffusion is described by the convectiondiffusion equation [21]:

$$
\frac{\partial m}{\partial t}=D \Delta m \pm r
$$

where $m$ is the molality of ions in aqueous solutions, $\mathrm{mol} \cdot \mathrm{kg}^{-1} ; t$ is time, $\mathrm{s} ; D$ is the diffusivity, $\mathrm{m}^{2} \cdot \mathrm{s}^{-1}$; and $r$ is the source or consumption rate term depending on the dissolution or crystallization kinetics, $\mathrm{mol} \cdot \mathrm{kg}^{-1} \cdot \mathrm{s}^{-1}$. In our work, the term $r$ means the coupling of the crystallization rate with the dissolution rate in crystallization or dissolution process; that is,

$$
r=A_{\mathrm{c}} \cdot(J-G),
$$

where $J$ and $G$ are dissolution and crystallization rates (mol $\cdot \mathrm{kg}^{-1} \cdot \mathrm{m}^{-2} \cdot \mathrm{s}^{-1}$ ), respectively. $J$ and $G$ are calculated based on chemical potential difference. $A_{\mathrm{c}}$ is the average area of the crystals, $\mathrm{m}^{2}$.

The dissolution kinetics of $\mathrm{KCl}$ at $298.15 \mathrm{~K}$ is investigated with ion selective electrodes and modified statistical rate theory [11,12].

For the crystallization process, three kinds of crystal growth mechanisms (spiral growth mechanism, twodimensional nucleation growth mechanism, and adhesivetype mechanism) have been proposed [14]. In our work, adhesive-type mechanism is selected because the fractional crystallization process of carnallite is very fast and it is reasonable to assume that the nucleation and crystal growth occur at the same time. Therefore, the crystallization rate is expressed as [14]

$$
G=\mathrm{A} \cdot\left(\frac{\Delta \mu}{R T}\right)=\mathrm{A} \ln \left(\frac{A_{\mathrm{sp}}}{K_{\mathrm{sp}}}\right),
$$

where A is a constant, $\mathrm{mol} \cdot \mathrm{kg}^{-1} \cdot \mathrm{m}^{-2} \cdot \mathrm{s}^{-1} ; \Delta \mu$ is the chemical potential difference, $\mathrm{J} \cdot \mathrm{mol}^{-1} ; R$ is the universal gas constant, $\mathrm{J} \cdot \mathrm{mol}^{-1} \cdot \mathrm{K}^{-1}$; and $T$ is temperature, $\mathrm{K}$.

The diffusion of $\mathrm{K}^{+}, \mathrm{Mg}^{2+}$, and $\mathrm{Cl}^{-}$and the crystallization kinetics of $\mathrm{KCl}$ in aqueous solutions are studied in detail ${ }^{1)}$. The results show that obvious fluctuations appear at $1200 \mathrm{~s}$ in the channel widths of $0.2 \mathrm{~m}$. The fluctuations of the $\Delta \mu$ of $\mathrm{KCl}$ around $\Delta \mu=0$ give us an explanation of 
how the porous structures form after carnallite dissolving in pure water; that is, when $\Delta \mu$ is positive, it means that the aqueous solution is supersaturated and crystallization may occur; when $\Delta \mu$ is negative, it means that the aqueous solution is unsaturated or the crystals that have been formed may dissolve again. The magnitude of $\Delta \mu$ for the dissolution of $\mathrm{KCl}$ is larger than that for the nucleation, and the differences between the two driving forces near the carnallite boundary bring the porous crystal structures. Moreover, the investigation also shows the importance of the ionic activity coefficient calculation for the investigated systems. This may provide a reference for other porous structure formation in the advanced material synthesis.

\section{Conclusions}

From the above introduction, we can draw the following conclusions:

1) The research framework for crystallization modeling has been proposed. If the investigations of the rigorous crystallization modeling by thermodynamics are required, four steps should be performed. The first is the activity coefficient model of the solution, the second is SLE, and the third and fourth are the dissolution and crystallization kinetics modeling, respectively.

2) By combining the dissolution and crystallization kinetics modeling, the mechanisms for complex structure formation and microphase transition can be analyzed.

Acknowledgements This work was supported by the Chinese National Key Technology Research and Development Program (Grant Nos. 2006BAB09B02, 2006AA03Z455, and 2006BAE03B7-2), the Chinese National Science Foundation for Outstanding Young Scholars (No. 20428606), the Natural Science Foundation of the Jiangsu Higher Education Institutions of China (08KJB530003), the National Natural Science Foundation of China (Grant Nos. 20676062, 20731160614, 20736002, B061101, B060105, 20706029, and 20706028), NSFC-RGC (No. 20731160614), the Key Science Foundation of Jiangsu Province, China (BK 2004215), and Program for Changjiang Scholars and Innovative Research Team in University (No. IRT0732).

\section{References}

1. Wei J. Product Engineering: Molecular Structure and Properties. New York: Oxford University Press, 2006

2. Li J H, Zhang J Y, Ge W, Liu X H. Multi-scale methodology for complex systems. Chemical Eng Sci, 2004, 59(8-9): 1687-1700

3. Xu N P, Shi J. Progress in material-oriented chemical engineering of China. Journal of Chemical Industry and Engineering (China), 2003, 54(4): 423-426 (in Chinese)

4. $\mathrm{Lu} \mathrm{X} \mathrm{H,} \mathrm{Maurer} \mathrm{G.} \mathrm{Model} \mathrm{for} \mathrm{describing} \mathrm{activity} \mathrm{coefficients} \mathrm{in}$ mixed electrolyte aqueous solutions. AIChE J, 1993, 39(9): 1527-
1538

5. Lu X H, Zhang L Z, Wang Y R, Shi J. Simultaneous prediction of activity coefficients and enthalpy for aqueous electrolyte solutions at high temperatures. Fluid Phase Equilibria, 1996, 116(1-2): 201-208

6. Lu X H, Zhang L Z, Wang Y R, Shi J, Maurer G. Prediction of activity coefficients of electrolytes in aqueous solutions at high temperatures. Ind Eng Chem Res, 1996, 35(5): 1777-1784

7. Ji X Y, Feng X, Lu X H, Zhang L Z, Wang Y R, Shi J. A generalized method for the solid-liquid equilibrium stage and its application in process simulation. Ind Eng Chem Res, 2002, 41(8): 2040-2046

8. Salem M R, Mangood A H, Hamdona S K. Dissolution of calcite crystals in the presence of some metal ions. J Mater Sci, 1994, 29 (24): 6463-6467

9. Palwe B G, Tavare N S. Growth kinetics of potassium sulphate crystals in a DTB agitated crystallizer. Chem Eng Sci, 1984, 39(5): 903-905

10. Kralj D, Brecevic L, Kontrec J. Vaterite growth and dissolution in aqueous solution. III: Kinetics of transformation. J Cryst Growth, 1997, 177(3-4): 248-257

11. Ji X Y, Chen D L, Wei T, Lu X H, Wang Y R, Shi J. Determination of dissolution kinetics of $\mathrm{K}_{2} \mathrm{SO}_{4}$ crystal with ion selective electrode. Chemical Engineering Science, 2001, 56(24): 7017-7024

12. Liu C, Feng X, Ji X Y, Chen D L, Wei T, Lu X H. The study of dissolution kinetics of $\mathrm{K}_{2} \mathrm{SO}_{4}$ crystal in aqueous ethanol solutions with a statistical rate theory. Chinese Journal of Chemical Engineering, 2004, 12(1): 128-130

13. Bergfors T. Protein Crystallization. California: International University Line, La Jooa, 1999

14. Mullin J W. Crystallization. Woburn: Butterworth-Heinnemann, 1997

15. Taguchi Y, Yoshida M, Kobayashi H J. Temperature dependence of the crystal growth rate of potassium sulfate at low temperature. Chem Eng Jpn, 2002, 35(11): 1038-1044

16. Feng X, Liu C, Ji X Y, Chen D L, Lu X H. The simulation and analysis for the process of potassium sulfate production by Glauber salt method. Gaoxiao Huaxue Gongcheng Xuebao, 2000, 14(6): 583-589 (in Chinese)

17. Kim S, Myerson A S. Metastable solution thermodynamic properties and crystal growth kinetics. Ind Eng Chem Res, 1996, 35(4): 1078-1084

18. Mohan R, Myerson A S. Growth kinetics: a thermodynamic approach. Chem Eng Sci, 2002, 57(20): 4277-4285

19. Cheng F Q, Bai Y, Liu C, Lu X H, Dong C. Thermodynamic analysis of temperature dependence of the crystal growth rate of potassium sulfate. Ind Eng Chem Res, 2006, 45(18): 6266-6271

20. Liu C, Ji Y H, Bai Y, Cheng F Q, Lu X H. Formation of porous crystals by coupling of dissolution and nucleation process in fractional crystallization. Fluid Phase Equilibria, 2007, 261(1-2): 300-305

21. Ji Y H, Ji X Y, Liu C, Lu X H. Modelling of mass transfer coupling with crystallization kinetics in microscale. Chem Eng Sci, 2009, 10.1016/j.ces.2009.12.045 